\title{
Regrowth-delay body as a bacterial subcellular structure marking multidrug- tolerant persisters
}

\author{
Jiayu Yu', Yang Liu', Huijia Yin ${ }^{1}$ and Zengyi Chang ${ }^{1,2}$
}

\begin{abstract}
Bacteria have long been recognized to be capable of entering a phenotypically non-growing persister state, in which the cells exhibit an extended regrowth lag and a multidrug tolerance, thus posing a great challenge in treating infectious diseases. Owing to their non-inheritability, low abundance of existence, lack of metabolic activities, and high heterogeneity, properties of persisters remain poorly understood. Here, we report our accidental discovery of a subcellular structure that we term the regrowth-delay body, which is formed only in non-growing bacterial cells and sequesters multiple key proteins. This structure, that dissolves when the cell resumes growth, is able to be viewed as a marker of persisters. Our studies also indicate that persisters exhibit different depth of persistence, as determined by the status of their regrowth-delay bodies. Our findings imply that suppressing the formation and/or promoting the dissolution of regrowth-delay bodies could be viable strategies for eradicating persisters.
\end{abstract}

\section{Introduction}

It has been well documented that, in a genetically homogeneous population of bacterial cells, a subset is able to enter a phenotypically dormant, non-growing (or, more precisely, low metabolic activity) state. This state has been connected to those named as sporulation, latency, regrowth lag, persisters, or the viable but nonculturable, in laboratory, clinical, or environmental microbiology ${ }^{1-7}$. Although this state of bacterial cells has been recognized for more than 100 years, much remain unknown on its properties, such as how the bacterial cells enter, maintain and exit such a unique state, that is best known for its non-inheritable multidrug tolerance ${ }^{4,8-11}$.

The regrowth lag phenomenon, initially recognized by Max Muller in 1895, was observed as soon as bacterial culturing became feasible ${ }^{12}$, but remains the most

\footnotetext{
Correspondence: Zengyi Chang (changzy@pku.edu.cn)

${ }^{1}$ The State Key Laboratory of Protein and Plant Gene Research, School of Life Sciences, Peking University, 100871 Beijing, China

${ }^{2}$ Center for Protein Science, Peking University, 100871 Beijing, China

These authors contributed equally: Jiayu Yu, Yang Liu, Huijia Yin
}

poorly understood stage of the bacterial growth cycle $^{6,13}$. In a related phenomenon, bacterial dormancy was defined as a state of certain bacterial cells that exhibits a long-lasting regrowth $\operatorname{lag}^{1,2}$. Later, the term persister was coined to denote an extremely small subpopulation of dormant, non-dividing bacterial cells that are not killed by concentrations of antibiotics sufficiently high to kill the actively dividing ones ${ }^{14}$. The persisters were presumed to be responsible for the post-treatment relapse of bacterial infections ${ }^{4,5,14-16}$. It was emphasized that the persisters are not resistant to antibiotics, since they produce offspring that are as susceptible to antibiotics as their parent cells ${ }^{14}$. More recently, it was unveiled that the bacterial cells in the natural environment are commonly in a viable but nonculturable dormant state ${ }^{17,18}$, one that is highly similar to the persisters.

Although much effort has been made to understand the molecular mechanisms leading to the formation of persisters, and certain specific protein factors (like the Hip) or small molecules (like the pppGpp) have been claimed to be important for this process ${ }^{19-21}$, not much is certain up

\section{(c) The Author(s) 2019}

(c) (i) Open Access This article is licensed under a Creative Commons Attribution 4.0 International License, which permits use, sharing, adaptation, distribution and reproduction in any medium or format, as long as you give appropriate credit to the original author(s) and the source, provide a link to the Creative Commons license, and indicate if changes were made. The images or other third party material in this article are included in the article's Creative Commons license, unless indicated otherwise in a credit line to the material. If material is not included in the article's Creative Commons license and your intended use is not permitted by statutory regulation or exceeds the permitted use, you will need to obtain permission directly from the copyright holder. To view a copy of this license, visit http://creativecommons.org/licenses/by/4.0/. 
to now ${ }^{8,9,22,23}$. The slow pace of learning about this state of bacterial cells is apparently attributed to the great technical difficulty of unequivocally identifying them, which are presumed to exist in extremely small numbers in a genetically uniform population, often with no significant morphological distinctions ${ }^{8-10}$. Because of this, persisters have been hitherto commonly perceived only on the basis of their lack of growth and multidrug tolerance. In particular, persisters have been conventionally detected by indirectly measuring the number of colonyforming units (CFUs) after treating the cell samples with a high concentration of a certain antibiotic ${ }^{24}$, or as cells that do not grow in the presence, but regrow after the removal, of antibiotics when monitored with a microfluidic device ${ }^{25}$.

We have been trying to explore proteins when they are present in living bacterial cells, as by performing protein photo-crosslinking analysis mediated by genetically introduced unnatural amino $\operatorname{acids}^{26,27}$. In one recent study, we examined the assembly patterns of the FtsZ protein, which plays an essential role by assembling into the Z-ring structure for each bacterial cell to divide into two via the cytokinesis process ${ }^{28-30}$, as well as for each mitochondrion $^{31}$ or chloroplast $^{32}$ to divide into two. In particular, we revealed hitherto unreported lateral interactions between the FtsZ protofilaments that are essential for FtsZ to assemble into the dynamic Z-ring structure in living bacterial cells ${ }^{33}$.

As an exciting byproduct of that study, we accidentally revealed the presence of a novel reversible subcellular structure that we named it as the regrowth-delay body. This structure is formed in non-growing late stationaryphase bacterial cells and sequesters multiple proteins essential for cell growth. Remarkably, the regrowth-delay bodies become dissolved when a bacterial cell exits the regrowth lag and resumes growth, meanwhile releasing the sequestered proteins for re-functioning. We also demonstrated that a higher degree of regrowth-delay body formation is correlated to a longer duration of regrowth lag as well as a higher level of antibiotic tolerance, not only in E. coli but also in two bacterial pathogens. Therefore, the regrowth-delay body not only acts as a unique and highly valuable biomarker for distinguishing the non-growing dormant persister cells from the actively growing non-persister cells, but also acts as a dynamic biological timer for bacterial cells to exit the regrowth lag. Our studies also indicate that each persister exhibits a particular depth of persistence, which seems to explain the long-observed heterogeneous nature of the persister subpopulation. Our findings should be proven greatly valuable not only for specifically identify and explore the persisters in any cell population, but also for designing viable strategies to eradicate the formidable multidrugtolerant pathogenic persisters.

\section{Results}

The cell division protein FtsZ no longer self-assembles but exists as an insoluble form in late stationary-phase bacterial cells

In an attempt to unveil how FtsZ assembles into the dynamic Z-ring structure during the cytokinesis of bacterial cell division, we performed systematic protein photo-crosslinking analyses with FtsZ variants containing the genetically introduced photoactive unnatural amino acid pBpa ( $p$-benzoyl-L-phenylalanine) ${ }^{34}$ in living E. coli cells. This allowed us to uncover novel lateral interactions between the FtsZ protofilaments that were demonstrated to be essential for cell division ${ }^{33}$.

During these studies, out of curiosity, we additionally examined the status of FtsZ in non-dividing/non-growing $E$. coli cells, as has never been addressed by people working with FtsZ. We revealed, as expected, that a pBpa variant of FtsZ, though self-assembled into homooligomers in actively dividing log-phase cells (Supplementary Fig. S1a, lanes 2 and 6), no longer does so (lanes 4 and 8 ) in the non-dividing/non-growing late stationaryphase cells (the technical details of these experiments are described in the legend of Supplementary Fig. S1). Astonishingly, we observed that most of the free FtsZ monomers, together with almost all the photo-crosslinked products, were detected in the insoluble pellet fraction of lysates of the late stationary-phase cells (Supplementary Fig. S1b, lane 8). By contrast, all the photo-crosslinked FtsZ dimers and the free FtsZ monomers were principally detected in the soluble supernatant fractions of lysates of the log-phase cells (lane 3).

In light of this puzzling observation, we then examined the distribution pattern of the endogenous FtsZ (instead of the FtsZ variant we examined above) in E. coli cells. Likewise, we revealed that the endogenous FtsZ protein was largely detected in the soluble supernatant fraction of log-phase cells (Fig. 1a, lane 2), but in the insoluble pellet fraction of late stationary-phase cells (lane 6). As comparison, we demonstrated that $\mathrm{EF}^{-} \mathrm{Tu}$ (one of the most abundant proteins in bacterial cells) and GroEL (a molecular chaperone binding to misfolded client proteins) were both largely detected in the supernatant fraction (Fig. 1a, lanes 2 and 5), with hardly any in the pellet fraction (lanes 3 and 6) of either log-phase or late stationary-phase cells. Taken together, these results revealed for the first time that the FtsZ protein (as well as proteins interacting with it) exists as an insoluble form in non-dividing/non-growing late stationary-phase bacterial cells.

\section{The FtsZ protein exists in two cell-pole granules in each late stationary-phase bacterial cell}

We subsequently tried to monitor the status of FtsZ by performing live-cell imaging analysis. For this purpose, we 


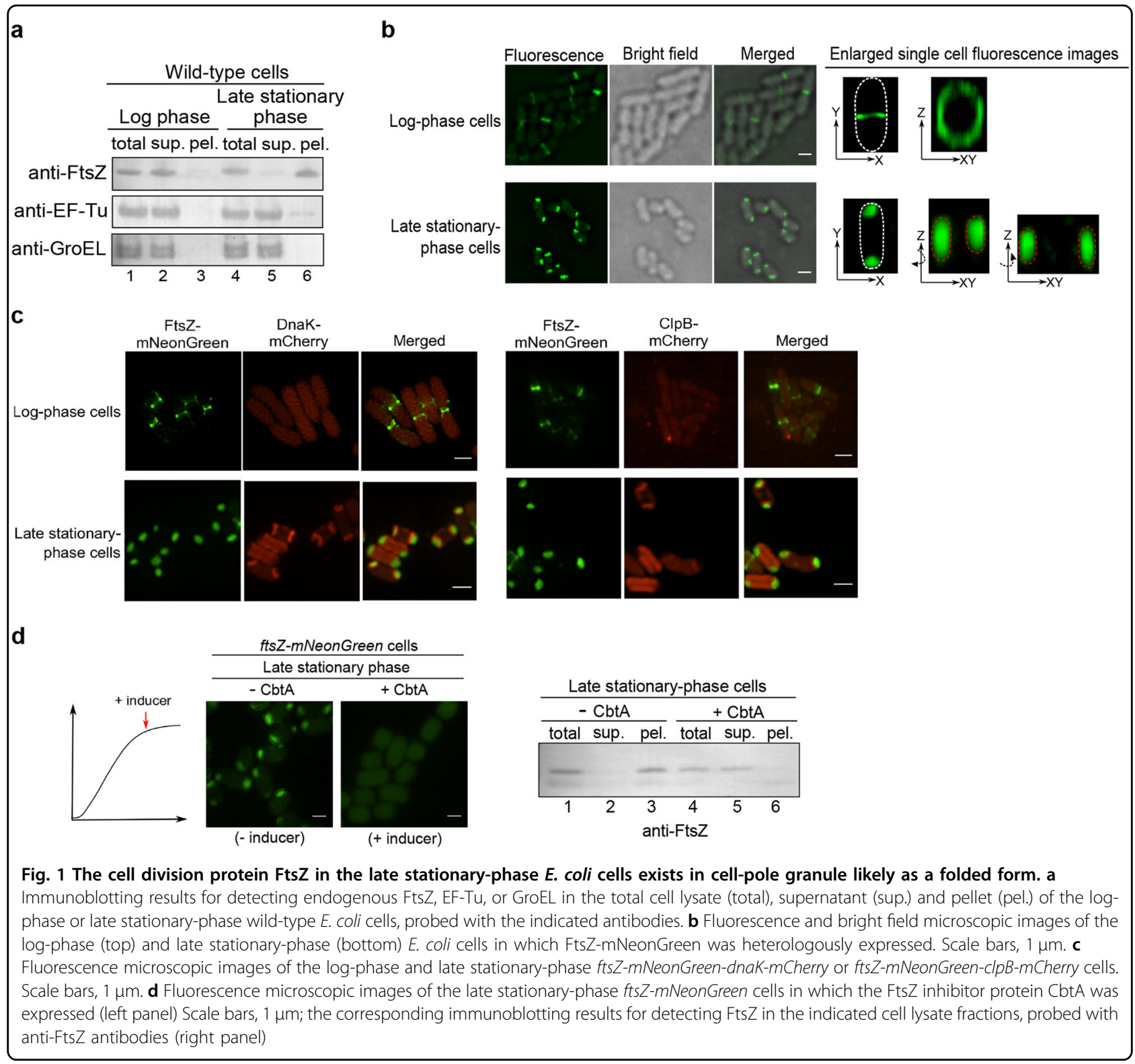

started by heterologously expressing FtsZ-mNeonGreen, a form of FtsZ being fused to the green fluorescent protein mNeonGreen, in E. coli cells. Here, the fusion protein was expressed at a relatively low level, which was achieved via the leaky transcription of the Tet promoter (i.e., with no addition of the inducing agent), such that the fluorescent FtsZ fusion protein would be incorporated into, but not interrupt, the Z-ring structure that was largely formed via the assembly of endogenous wild-type FtsZ. We first verified an effective incorporation of FtsZ-mNeonGreen into the Z-ring in log-phase cells (Fig. 1b, top), like what was reported before ${ }^{35}$. Remarkably, we then detected FtsZ$\mathrm{mNeonGreen} \mathrm{as} \mathrm{two} \mathrm{cell} \mathrm{pole-granules} \mathrm{in} \mathrm{each} \mathrm{late}$ stationary-phase cell (Fig. 1b, bottom). As a control, the unfused fluorescent mNeonGreen protein was shown to be evenly distributed in the cytoplasm of either activelydividing or non-dividing bacterial cells (Supplementary Fig. S2a). Furthermore, the immunofluorescent analysis ${ }^{36}$ also illustrated that the endogenous FtsZ proteins indeed exist as the form of cell-pole granules in the late stationaryphase cells (Supplementary Fig. S2b).

For further systematic live-cell imaging analysis, we subsequently constructed a bacterial strain whose genome was modified to express FtsZ-mNeonGreen (rather than from a plasmid), in parallel with the normally expressed endogenous FtsZ. In particular, we integrated the ftsZ-mNeonGreen gene into the genomic rhamnose operon (as illustrated in Supplementary Fig. S3a) and demonstrated that the FtsZ-mNeonGreen protein would be produced only in the presence of rhamnose 
(the inducing sugar) in this ftsZ-mNeonGreen strain (Supplementary Fig. S3b), hardly affecting the growth of the cells (Supplementary Fig. S3c). We also verified the presence of FtsZ-mNeonGreen in the Z-ring structure in log-phase but in the cell-pole granules in late stationaryphase ftsZ-mNeonGreen cells (Supplementary Fig. S3d).

Our live-cell imaging analysis employing this ftsZ$m N e o n G r e e n$ strain revealed that the cell-pole granules seem to be closely associated with the inner membrane but not surrounded by it (Supplementary Fig. S4a), as verified by results (Supplementary Fig. S4b) of staining with the membrane-specific dye FM4-6 $4^{37}$. These imaging results meanwhile demonstrated that the cell-pole granules occupy cytosolic locations that are hardly accessible to other cytosolic proteins (Supplementary Fig. S4a, bottom panel), suggesting a compact nature. In line with this, we observed that these granules were maintained intact even after the cells were broken (Supplementary Fig. S4c).

\section{The FtsZ protein in cell-pole granules are likely folded}

Aggregates of misfolded proteins have been reported to exist at the poles in E. coli cells under heat shock conditions $^{38,39}$. Additionally, insoluble proteins, which were naturally assumed to be misfolded, have been reported to accumulate in stationary-phase E. coli cells ${ }^{40-42}$. In view of these reports, we then attempted to clarify the folding status of FtsZ in the cell-pole granules, despite the fact that FtsZ was demonstrated to exist in a soluble form when heterologously over-expressed in bacterial cells ${ }^{43}$.

Considering that the molecular chaperones DnaK and $\mathrm{ClpB}$, as well as the protease $\mathrm{ClpP}$ were reported to be associated with protein aggregates formed under stress conditions $^{39}$, we decided to analyze whether or not they are associated with the cell-pole granules. Our blotting analysis demonstrated that all these three quality control proteins were primarily detected in the supernatant (Supplementary Fig. S5a, lane 2) with hardly any detected in the pellet (lane 3 ) of late stationary-phase cell lysates. In line with this, our live-cell imaging data showed that neither DnaK nor $\mathrm{ClpB}$, each being expressed as a form fused to the red fluorescent protein mCherry (by manipulating their endogenous genes on the genomic DNA of the ftsZ-mNeonGreen strain), was detected in the cell-pole granules (Fig. 1c). The imaging data meanwhile revealed, interestingly, that both DnaK and ClpB, though being evenly dispersed in the cytosol of log-phase cells, were concentrated near the two cell poles, at sites very close to but clearly separate from the FtsZ-containing cell-pole granules, but only in a small number of late stationaryphase cells (Fig. 1c). These subcellular sites, which might represent ones where DnaK and $\mathrm{ClpB}$ (themselves being in soluble forms, as shown in Supplementary Fig. S5a) were co-localized with certain form of protein aggregates, are worth further investigation in the future. Taken together, these results did not provide evidence to support the possibility that the cell-pole granules are typical aggregates formed by misfolded proteins.

As an attempt to further assess the folding status of FtsZ in the cell-pole granules, we examined whether inhibitor proteins that specifically bind to folded FtsZ could prevent FtsZ from entering the granules. For this purpose, we analyzed the CbtA and KilR proteins, each of which was known to bind to and to block monomeric FtsZ for assembling into the Z-ring in cells ${ }^{44,45}$. Either CbtA or KilR was then expressed from a plasmid, under the control of an anhydrotetracycline-inducible promoter. We first verified their capacity to inhibit FtsZ from assembling into the Z-ring in log-phase ftsZ-mNeonGreen cells (Supplementary Fig. S5b).

We then showed that FtsZ was no longer able to enter the cell-pole granules when the CbtA expression was induced at the stationary phase (Fig. 1d, left panel). In agreement with these findings, our immunoblotting analysis confirmed that FtsZ became undetectable in the pellet fraction but remained in the supernatant when CbtA was expressed (Fig. 1d, right panel). It should be mentioned that we failed to observe any effect of KilR on the inhibition of cell-pole granule formation (Supplementary Fig. S5c). Possible explanations for these observations include that the level of KilR was not high enough, or its binding mechanism did not interrupt the formation of cell-pole granules.

Furthermore, the conclusion that FtsZ in the cell-pole granules is folded was also supported by our in vivo protein photo-crosslinking analysis. Specifically, the data (Supplementary Fig. S5d) reveal that when the unnatural amino acid residue $\mathrm{pBpa}$ was placed at residue positions close to each other in space (e.g., positions 151, 166, and 174 , or $31,47,51$, and 54) according to the reported crystal structure ${ }^{46}$, similar patterns of photo-crosslinked products were generated. By contrast, when $\mathrm{pBpa}$ was placed at sites that were spatially distant (e.g., positions $61,85,299$, and 340), different patterns of photocrosslinked products were detected.

Collectively, these results seem to indicate that FtsZ in the granules is folded, rather than misfolded.

\section{The cell-pole granules become dissolved in cells exiting their regrowth lag and resuming growth}

We next sought to decipher the fate of the cell-pole granules when the bacterial cells resume their growth. For this purpose, we re-cultured the non-growing late stationary-phase ftsZ-mNeonGreen cells in fresh culture medium lacking the inducer rhamnose to avoid the production of new FtsZ-mNeonGreen protein. Remarkably, we observed an effective relocation of FtsZ-mNeonGreen from the cell-pole granules to the Z-ring that was formed in cells ending their regrowth lag and resuming growth 
a

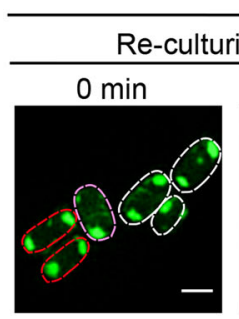

C

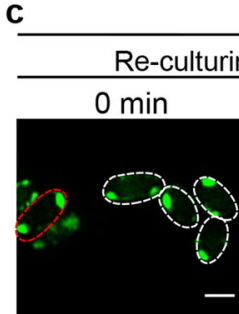

ftsZ-mNeonGreen cells

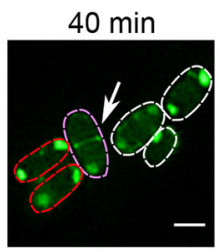

ftsZ-mNeonGreen cells
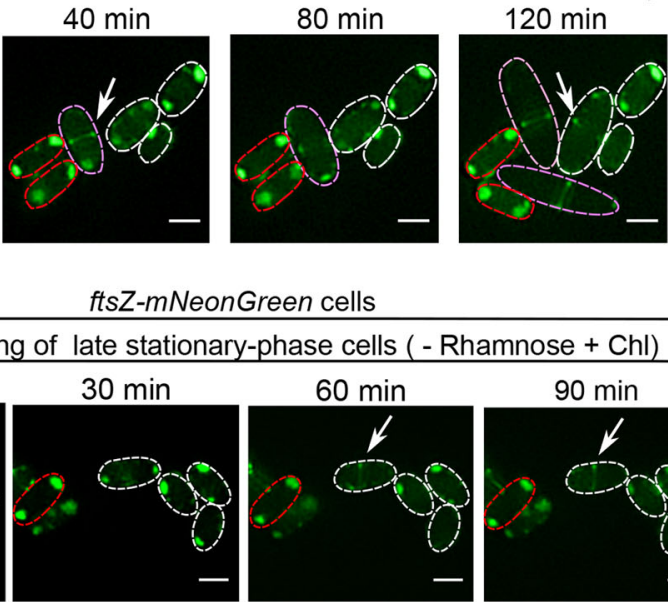
$60 \mathrm{~min}$

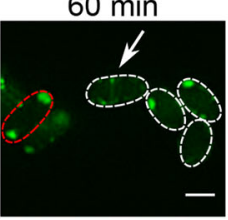

b

Late stationary-phase cells re-cultured to log phase

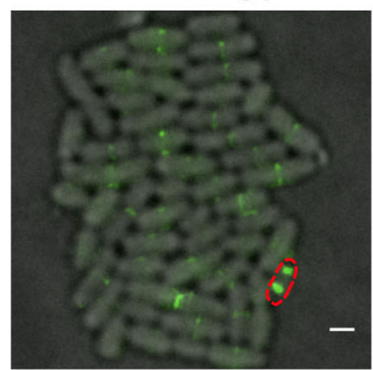

Fig. 2 When the non-growing cells exit their regrowth lag, the cell-pole granules dissolve to release the FtsZ for re-functioning, but maintain unaltered otherwise. a Fluorescence microscopic images of the re-cultured late stationary-phase ftsZ-mNeonGreen cells present in fresh LB medium lacking rhamnose, as obtained at the indicated time points. Note: one of the examined cells divided into two daughter cells at 120 min (circled by pink dashed lines). Scale bars, $1 \mu \mathrm{m}$. b Fluorescence microscopic images of the late stationary-phase ftsZ-mNeonGreen cells re-cultured to the log phase $\left(\mathrm{OD}_{600} \sim 0.5\right)$ in liquid LB medium lacking rhamnose. Scale bar, $1 \mu \mathrm{m}$. c Fluorescence microscopic images of the late stationary-phase ftsZ-mNeonGreen cells re-cultured to the indicated time points in fresh LB medium that lacked rhamnose and contained the antibiotic chloramphenicol. Scale bars, $1 \mu \mathrm{m}$

(Fig. 2a, exemplified by the cells circled with pink or white lines). These newly assembled Z-ring structures seemed to be fully functional since they enabled the mother cells to split into two daughter cells (e.g., the cell circled with dashed pink lines at $80 \mathrm{~min}$ divided into two daughter cells at $120 \mathrm{~min}$ ). Of equal importance, cells that remained in the regrowth lag state all retained their cell-pole granules (Fig. 2a, exemplified by the cells circled with red dashed lines).

Worth of high attention, when the late stationary-phase ftsZ-mNeonGreen cells were re-cultured in fresh liquid medium lacking rhamnose to the log phase (with an $\mathrm{OD}_{600}$ of $\sim 0.5$ ), we observed the maintenance of the cellpole granules in an extremely small number of cells (as represented by the cell circled with red lines in Fig. 2b), with all other cells being actively dividing. In our opinion, there is little doubt that such an inert cell, which is tolerant to antibiotics (as to be shown in Fig. 3f), might be the long-searched and elusive persisters ${ }^{14}$.

To assess whether such dissolution of the cell-pole granules could occur in the absence of any new synthesis of proteins in the cells, we repeated the above analysis by adding chloramphenicol, a ribosome-binding antibiotic that is known to inhibit protein synthesis in bacterial cells, to the fresh culture medium. Interestingly, we still observed an effective dissolution of the cell-pole granules in several cells exiting the regrowth-lag (Fig. 2c, cells circled with white dashed lines), and we even occasionally observed the re-formation of Z-ring in certain cells (as indicated by the arrow). Of note, here the Z-ring would have to be formed mainly by using the FtsZ stored in and released from the cell-pole granules, but fluorescently labeled by a small amount of the incorporated FtsZmNeonGreen ${ }^{35}$, also released from the granules. We again observed the cell-pole granules to be retained in some other cells (Fig. 2c, exemplified by the cell circled with red dashed lines). In agreement with these live-cell imaging data, our immunoblotting analysis verified a timedependent decrease of FtsZ in the insoluble pellet (Supplementary Fig. S6, lanes 3, 6, and 9), with a corresponding increase of FtsZ in the soluble supernatant (lanes 2, 5, and 8) when the wild-type late stationaryphase cells were re-cultured in fresh medium containing chloramphenicol.

Importantly, the results displayed in Fig. 2 also revealed that the cell-pole granules present in different individual cells seem to exhibit a high degree of heterogeneity. Specifically, the cell-pole granules became totally dissolved, partially dissolved, or remained almost completely unaltered depending on the particular cell (clearly shown by the cells viewed at $120 \mathrm{~min}$ in Fig. 2a as well as those at $90 \mathrm{~min}$ in Fig. 2c). These data meanwhile suggest that an effective dissolution of the cell-pole granules is accompanied with a cell to end its regrowthlag and resume growth, whereas the lack of their dissolution may mark the maintenance of the non-growing 
a

ftsZ-mNeonGreen cells (from log phase to late stationary phase)

b

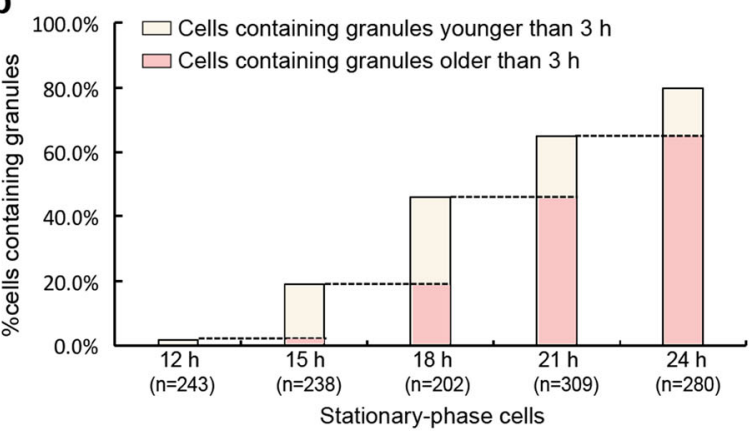

d

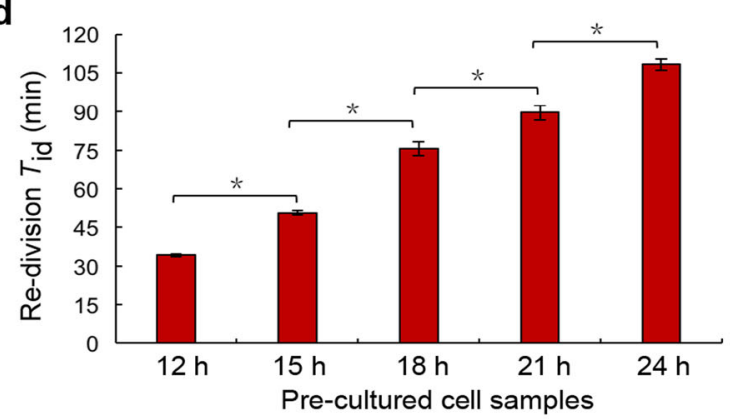

C

No. of cells retaining granules longer than $30 \mathrm{~min}$ total No. of granule-containing cells

No. of cells containing granules older than $3 \mathrm{~h}$ total No. of granule-containing cells

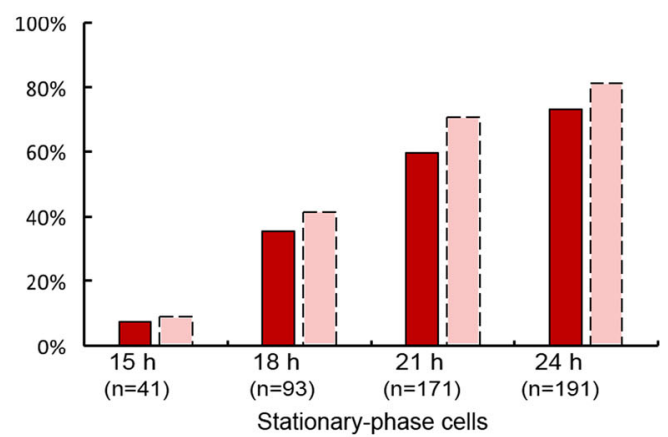

e
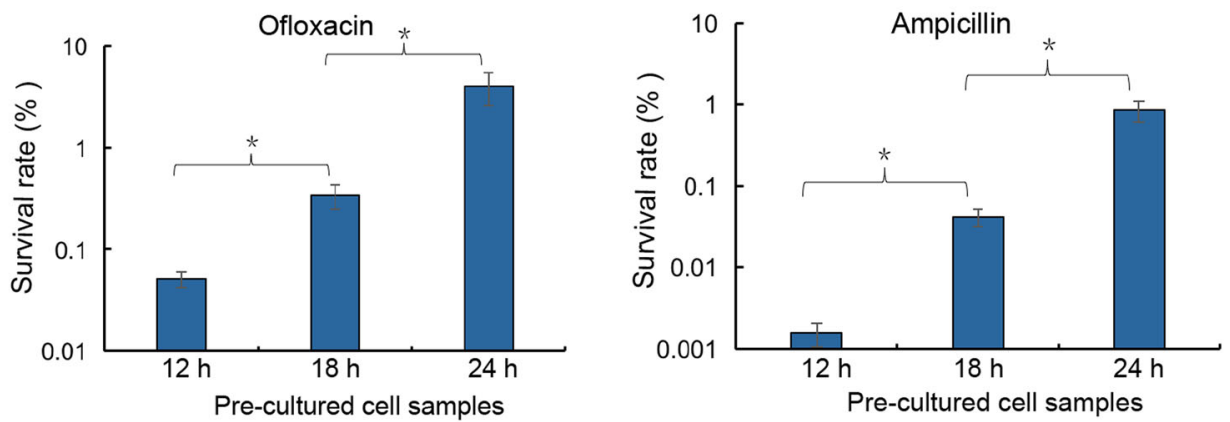

f

ftsZ-mNeonGreen Cells

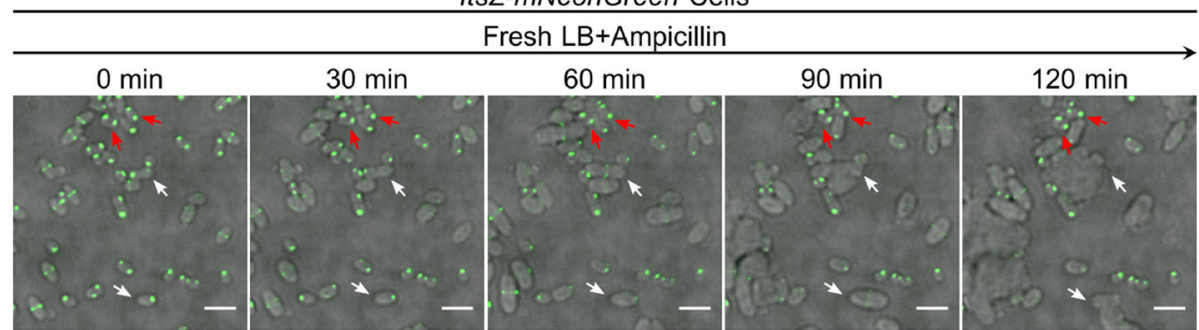

Fig. 3 (See legend on next page.) 
(see figure on previous page)

Fig. 3 The regrowth-delay bodies are formed heterogeneously in the cell population and the degree of their formation correlates with the duration of regrowth lag and the level of multidrug tolerance. a Fluorescence microscopic images of ftsZ-mNeonGreen cells cultured to the indicated time points in LB medium containing $0.02 \%$ rhamnose (to induce the production of FtsZ-mNeonGreen). Scale bars, $1 \mu \mathrm{m}$. $\mathbf{b}$ Percentage of cells containing regrowth-delay bodies that were cultured to the indicated time points. $\mathbf{c}$ Percentages of cells retaining their regrowth-delay bodies when the particular stationary-phase cell samples were re-cultured for $30 \mathrm{~min}$ in fresh medium containing chloramphenicol (red columns). Percentages of cells containing regrowth-delay bodies for more than $3 \mathrm{~h}$ (pink columns) were calculated from Fig. 3b. $\mathbf{d}$ Re-division $T_{\text {id }}$ (the average initial doubling time) values of wild-type cells that were pre-cultured to the indicated time points. The $T_{\text {id }}$ values were calculated based on the increase in cell numbers within the first $30 \mathrm{~min}$ of re-culturing (after diluting 40 -fold) in fresh medium at $37^{\circ} \mathrm{C}$ (for details, see Methods). e Survival rates of the indicated re-cultured stationary-phase wild-type cells that were treated with ofloxacin $(5 \mu \mathrm{g} / \mathrm{ml})$ or ampicillin $(200 \mu \mathrm{g} / \mathrm{ml})$ for $2 \mathrm{~h}$ (in fresh $\mathrm{LB}$ medium at $37^{\circ} \mathrm{C}$ ). The survival rates were calculated according to the equation: [colony-forming units (CFU) of the antibiotic-treated cells]/[colonyforming units of the untreated cells] $\times 100$. $\mathbf{f}$ Live-cell fluorescence microscopic images merged with bright field of the re-cultured late stationaryphase ftsZ-mNeonGreen cells in the fresh ampicillin-containing LB medium (at $37^{\circ} \mathrm{C}$ ), as obtained at the indicated time points. Representative cells that exited (eventually became lysed) or maintained (unaltered) the regrowth lag is indicated by the white or red arrows, respectively. Scale bars, 3 $\mu \mathrm{m}$. The symbol * in $\mathbf{d}$ and $\mathbf{e}$ denotes a significant difference between the compared pair of samples $(P$-value $<0.05, t$-test). At least three biological replicates were analyzed in obtaining each value

persister state for a cell (as represented by the one shown in Fig. 2b).

\section{The cell-pole granules are formed in a highly heterogeneous manner in different individual cells and in a progressive manner in each cell}

We next attempted to learn more about the nature of the manifested heterogeneity of the cell-pole granules (as shown in Fig. 2), by examining their formation process in the non-growing bacterial cells. For this purpose, we initially planned to employ a microfluidic chip device to monitor both the formation, during the non-growing phase, and the dissolution, during the regrowth phase, of the cell-pole granules in single ftsZ-mNeonGreen cells. Although we observed the dissolving process of the cellpole granules, unfortunately, we were unable to set up a culturing condition under which the cell-pole granules were formed in the bacterial cells in the available microfluidic system. Given this failed attempt, we then decided to address this issue by analyzing the cell population.

We started by conducting a qualitative live-cell imaging analysis to assess how cell-pole granules are formed in the non-growing ftsZ-mNeonGreen cells along the culturing process. The data, displayed in Fig. 3a, revealed that the formation of cell-pole granules appears to occur in a progressive manner in each individual cell, as the sizes of the cell-pole granules appeared to be different in different individual cells. Meanwhile, the data indicates a high heterogeneity in regards to the formation of cell-pole granules among the cell population. For instance, at $15 \mathrm{~h}$ of culturing, cell-pole granules are formed in some of the cells, while a small portion of other cells (indicated by the red arrow) were still dividing (i.e., with Z-ring remained visible). Subsequently, we performed a quantitative livecell imaging analysis to calculate the percentage of cells in which cell-pole granules were formed at the different culturing time points. As displayed in Fig. $3 \mathrm{~b}$, the percentage of cells containing the cell-pole granules clearly increased along the culturing process. Together, these results indicate that the cell-pole granules are formed in a highly heterogeneous manner among the individual bacterial cells and in a progressive fashion in each individual cell.

\section{Bacterial cells containing more aged cell-pole granules stay in their regrowth-lag state for longer duration}

To uncover the potential relationship between the forming degree of cell-pole granules during stationary phase and the timing of their dissolution during reculturing for individual cell, we then measured the percentage of cells in which cell-pole granules were formed at different culturing time points that still retained their cellpole granules after being re-cultured in fresh medium for $30 \mathrm{~min}$. The data, shown in Fig. 3c (red columns) clearly indicate that a higher percentage of cells retained their cell-pole granules when the non-growing cell samples were taken from a later stationary phase culturing point. We also noticed a significant correlation between the percentages of cells retaining their cell-pole granules for longer than $30 \mathrm{~min}$ during the re-culturing and the percentages of cells whose cell-pole granules are older than 3 $\mathrm{h}$ as formed during the stationary phase (pink columns in Fig. 3c, calculated based on the data presented in Fig. 3b). These data suggest that the more aged cell-pole granules are retained for a longer term during re-culturing.

The correlation between the duration of regrowth-lag and the age of cell-pole granules was further demonstrated by comparing the average re-division initial doubling times (re-division $T_{\mathrm{id}}$ ) manifested by the cells that were taken from different stationary-phase culturing time points and re-cultured. Here, for each cell sample, the redivision $T_{\text {id }}$ value was calculated based on its re-culturing growth curve (as displayed in Supplementary Fig. S7), and reflects its duration of regrowth-lag. The data, presented 
in Fig. 3d, clearly reveal a higher re-division $T_{\text {id }}$ value for a cell sample taken from a later culturing time point in the stationary phase. Collectively, these results suggest that for each bacterial cell, the duration of its regrowth-lag is apparently related to the status of its cell-pole granules. In light of this, we hereafter designate the cell-pole granule as the regrowth-delay body.

\section{Bacterial cells containing the regrowth-delay bodies are multidrug tolerant}

We then assessed whether bacterial cells that contain regrowth-delay bodies are tolerant to multiple antibiotics, being a major feature attributed to persisters. To this end, we first compared the antibiotic tolerance capacity of the non-growing cells taken from different stationary-phase culturing time points. The data clearly show that the bacterial cells derived from a later culturing point, thus possessing a higher level of aged regrowth-delay bodies, exhibited a significantly higher level of tolerance towards the two examined antibiotics, either ofloxacin or ampicillin (Fig. 3e). More importantly, our live-cell imaging data provide direct evidence showing that the bacterial cells retaining their regrowth-delay bodies would effectively survive the ampicillin treatment during the reculturing process (as represented by the cell labeled by red arrows in Fig. 3f). By contrast, the cells having their regrowth-delay body dissolved would be efficiently killed (eventually lysed) under the same re-culturing condition (as represented by the cell labeled by white arrows in Fig. 3f). The data displayed in Supplementary Fig. S8 also demonstrate that, without exception, the cells retaining their regrowth-delay bodies are tolerant to, thus are able to be enriched by ampicillin.

Collectively, our observations, as shown in Figs. 2 and 3, strongly suggest that the regrowth-delay bodies serve as effectively markers for the non-growing and antibiotictolerant bacterial persisters. It follows that the presence of regrowth-delay bodies would help us to efficiently identify the tiny subpopulation of persisters present in a large population of actively growing bacterial cell (as exemplified by the data shown in Fig. 2b). Our data meanwhile implicate that persister cells are probably in different depth of persistence depending on the age of their regrowth-delay bodies.

\section{The formation of regrowth-delay bodies selectively sequesters multiple key proteins}

We next attempted to characterize the composition of the regrowth-delay bodies to learn more about the properties of bacterial persisters. For this goal, we first tried to identify the proteins that are photo-crosslinked to multiple $\mathrm{pBpa}$ variants of FtsZ in the late stationary-phase cells. Specifically, we purified the photo-crosslinked products of five FtsZ variants with pBpa introduced at residue position 140 (lane 8 in Supplementary Fig. S1b), 47, 51, 61 or 166 (lane 10, 12, 16 or 4, respectively, in Supplementary Fig. S5d), each representing a different pattern of photocrosslinked products, by affinity chromatography via the Avi tag fused to FtsZ. The proteins were identified via mass spectrometry analysis and are listed in Supplementary Fig. S9a.

In light that intact regrowth-delay bodies were present in the pellet fraction (as shown in Supplementary Fig. S4c), we also performed mass spectrometry analysis on the collected pellet of the lysed wild-type $E$. coli cells, with the major proteins identified being also listed in Supplementary Fig. S9a. A functional annotation of these identified proteins revealed their key roles in cell growth (such as translation, transcription and metabolism) and division. Of note, some of the proteins (colored blue in Supplementary Fig. S9a) were identified by both mass spectrometry analyses.

We subsequently performed experiments to verify the presence of some of these identified proteins (other than FtsZ) in the regrowth-delay bodies. We first confirmed by live-cell imaging analysis that ZapC and FtsA (each being fused to $\mathrm{mNeonGreen),} \mathrm{two} \mathrm{additional} \mathrm{cell} \mathrm{division} \mathrm{pro-}$ teins identified, were both clearly detected in the regrowth-delay bodies as present in late stationary-phase cells, though in the Z-ring in log-phase cells (Fig. 4a). We also demonstrated that FtsA (as fused to the red fluorescent protein mCherry) co-localizes with FtsZ in the regrowth-delay bodies present either in living cells or in the lysates (Supplementary Fig. S9b). By contrast, FtsL and ZapA, two non-identified cell division proteins, were neither detected in the regrowth-delay bodies, while clearly detected in the Z-ring in log-phase cells (Supplementary Fig. S9c).

In addition to cell division proteins, we then verified the presence of five more identified ones. In particular, they, each being expressed as an Avi-tagged form and under the control of a constitutive promoter, were detected to a significant degree in the insoluble pellet fraction of lysates of late stationary-phase cells, though largely present in the supernatant of lysates of log-phase cells (Fig. 4b). Interestingly, among these five proteins, the three that were known to be essential for cell growth (i.e., ribosomal protein S5, tryptophan-tRNA ligase and transcriptional factor sigmaS) were almost fully detected in the pellet fraction (Fig. 4b). Of note, the sigmaS protein is known to be degraded in log-phase cells and accumulates only in stationary-phase cells ${ }^{47}$. Meanwhile, fluorescent imaging analysis of pyruvate dehydrogenase E1 and tryptophantRNA ligase, each being expressed as a fusion protein with mCherry in ftsZ-mNeonGreen cells, confirm their colocalization with FtsZ in the regrowth-delay bodies (Supplementary Fig. S9d). Taken together, these protein characterization and verification studies seem to suggest 

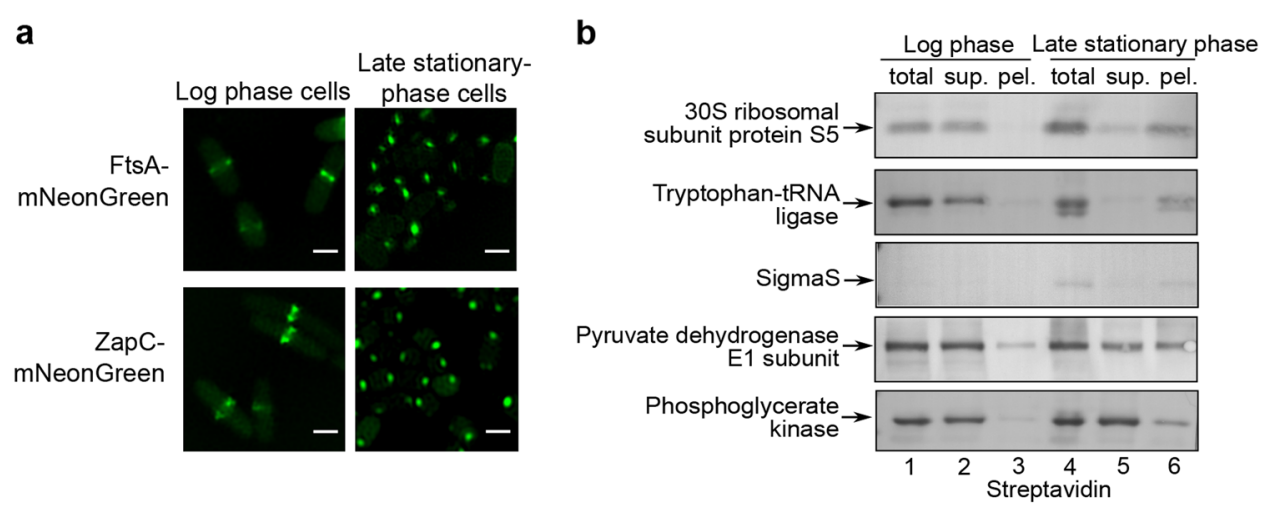

C

FtsA-mNeonGreen cells

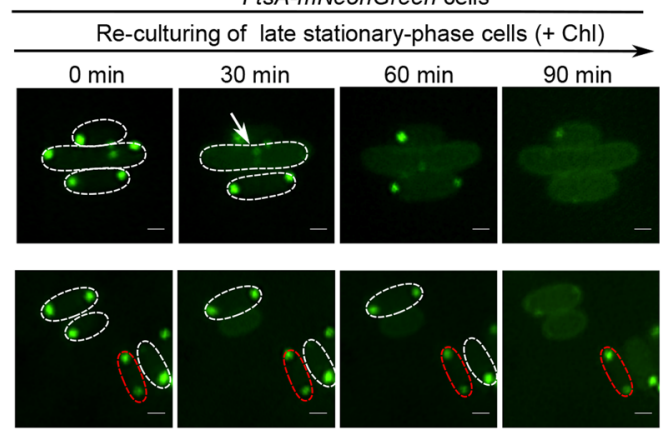

Fig. 4 Regrowth-delay bodies selectively sequester multiple key proteins that are released to re-function when cells exit their regrowth lag and resume growth. a Fluorescence microscopic images of the log-phase (left) and late stationary-phase (right) E. coli cells in which mNeonGreen-fused FtsA or ZapC (both being identified in the regrowth-delay bodies by mass spectrometry analysis, as shown in Supplementary Fig. S9a) was expressed from a plasmid under the control of a constitutive promoter. Scale bars, $1 \mu \mathrm{m}$. b Blotting results to analyze the indicated Avitagged proteins in the indicated lysate fractions of the log-phase or late stationary-phase wild-type cells, probed with streptavidin-AP. $\mathbf{c}$ Fluorescence microscopic images of the re-cultured late stationary-phase cells in which FtsA-mNeonGreen was expressed from a plasmid under the control of a constitutive promoter in fresh medium containing chloramphenicol, obtained at the indicated time points. Scale bars, $1 \mu \mathrm{m}$

that the regrowth-delay bodies sequester multiple important proteins that function in cell growth and division, which in turn may keep the cells in the non-growing persister state.

Additionally, we demonstrated by performing live-cell imaging analysis that similar to FtsZ, FtsA was also reutilized in cells exiting the regrowth-lag and resuming growth (Fig. 4c). Specifically, the FtsA protein (fused to $\mathrm{mNeonGreen)} \mathrm{either} \mathrm{reappeared} \mathrm{in} \mathrm{the} \mathrm{Z-ring} \mathrm{(shown} \mathrm{by}$ the arrow in Fig. 4c) of cells that were in the process of redividing or in the inner membrane of cells whose regrowth-delay bodies were dissolved but not yet dividing when the late stationary-phase cells were re-cultured in fresh medium containing chloramphenicol. Similarly, the FtsA protein was retained in the regrowth-delay bodies for cells remaining in the non-growing regrowth-lag state (represented by the cell circled by red dashed lines). These results once again demonstrated that the proteins sequestered in the regrowth-delay bodies could be released to resume their functions in cells exiting the regrowth-lag state and resuming growth.
Mutant bacterial cells with a reduced formation of regrowth-delay bodies exhibit a shorter duration of regrowth lag and a lower tolerance to antibiotics

To further examine the relationship between regrowthdelay body formation and regrowth lag time or antibiotic tolerance, we then attempted to generate mutant bacterial cells in which the formation of regrowth-delay bodies would be significantly reduced. Toward this goal, we referred to the list of proteins identified in the regrowthdelay bodies (as shown in Supplementary Fig. S9a) and realized the presence of multiple subunits of the respiratory chain complexes. Furthermore, our live-cell imaging analysis (Supplementary Fig. S4a) showed an apparent association of the regrowth-delay bodies with the inner membrane, where the respiratory chain complexes are located. In light of these observations, we then performed gene knockdown (or knockout) experiments to decrease or remove certain subunits of the respiratory chain complexes and analyzed whether the regrowth-delay body formation in the bacterial cells was significantly reduced. In particular, the nuo $A$ gene (encoding a subunit of 

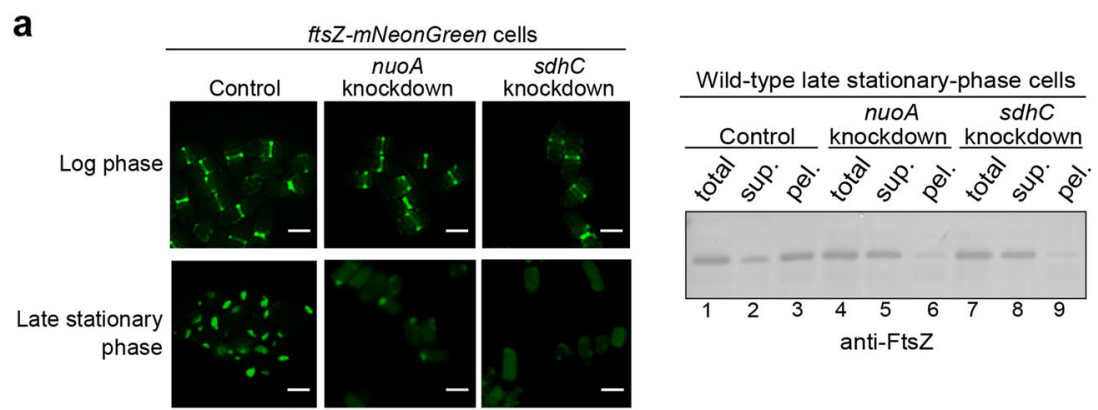

b

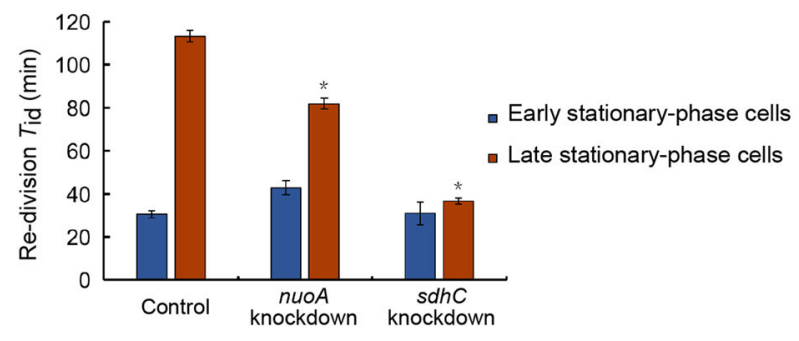

C

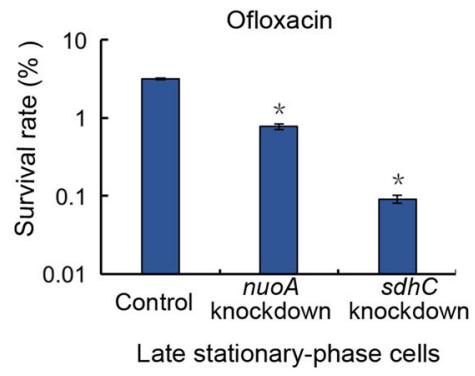

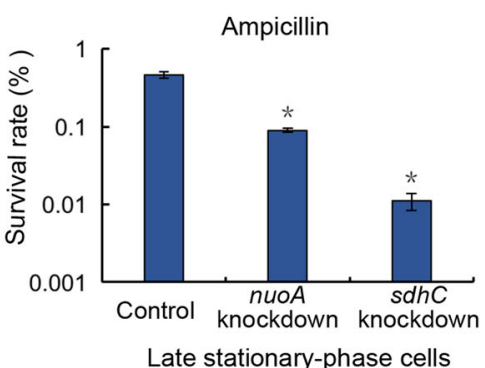

Late stationary-phase cells

Fig. 5 Mutant bacterial cells with a reduced formation of regrowth-delay bodies exhibit a shorter duration of regrowth lag as well as a lower tolerance to antibiotics. a Fluorescence microscopic images of the log-phase or late stationary-phase ftsZ-mNeonGreen cells having a knockdown of either the nuoA or the $s d h C$ gene. Cells expressing a non-targeting CRISPR RNA were analyzed as the control. Scale bars, $1 \mu \mathrm{m}$ (left panel). The immunoblotting results for detecting FtsZ in the indicated cell lysate fractions, as probed with anti-FtsZ antibodies (right panel). $\mathbf{b}$ Redivision $T_{\text {id }}$ values of early (blue bars; cultured to $12 \mathrm{~h}$ ) or late (red bars; cultured to $24 \mathrm{~h}$ ) stationary-phase cells of the indicated gene-knockdown strain. Here wild-type cells in which a non-targeting crRNA was expressed from a plasmid were analyzed as the control. c Survival rates of the late stationary-phase wild-type (control), nuoA-knockdown or sdhC-knockdown cells that were re-cultured in fresh medium after being treated with ofloxacin $(5 \mathrm{\mu g} / \mathrm{ml})$ or ampicillin $(200 \mu \mathrm{g} / \mathrm{ml})$. The survival rates were calculated according to the equation: (CFU of the antibiotic-treated cells)/(CFU of the untreated cells) $\times 100$. The symbol ${ }^{*}$ in $\mathbf{b}$ and $\mathbf{c}$ denotes a significant difference between the compared pair of samples $(P$-value $<0.05, t$-test). At least three biological replicates were analyzed for obtaining each value

respiratory chain complex I) or the $s d h C$ gene (encoding a subunit of respiratory chain complex II) in the ftsZmNeonGreen cells was subjected to knockdown manipulation using the CRISPRi technology ${ }^{48}$.

Our live-cell imaging analysis (Fig. 5a, left panel) demonstrated that the regrowth-delay body formation was significantly reduced in the late stationary-phase $n u o A$-knockdown cells and barely occurred in the $s d h C$ knockdown cells. In agreement with these imaging results, our immunoblotting analysis confirmed a significantly reduced amount of the endogenous FtsZ in the insoluble lysate pellet fraction of these cells, instead, much appeared in the soluble supernatant fraction (Fig. 5a, right panel). We observed similar reduction in regrowth-delay body formation (shown in Supplementary Fig. S10a) for the cells in which the nouAB (genes encoding two subunits of respiratory chain complex I) or $s d h C D A B$ (genes encoding all the four subunits of the respiratory chain complex II) were knocked out. Taken together, these observations indicate that the respiratory chain complexes somehow do play an important role for the formation of regrowthdelay bodies.

Furthermore, we observed that the re-division $T_{\text {id }}$ value of either the nuoA or $s d h C$ knockdown cells was significantly lower in comparison with that of the control cells (Fig. 5b). Additionally, the re-division $T_{\text {id }}$ values became comparable for the early and late stationary-phase $s d h C$ knockdown cells. Consistently, the survival rates of 
these non-growing late stationary-phase cells became significantly lower than those of the control cells after being treated with an antibiotic, ofloxacin or ampicillin (Fig. 5c). These observations on the gene knockdown cells further strengthened our conclusion that the formation of regrowth-delay bodies is correlated with the regrowth lag and antibiotic tolerance.

\section{Regrowth-delay body formation occurs in pathogenic bacteria and also correlates to the regrowth-lag and multidrug tolerance}

We subsequently demonstrated the formation of regrowth-delay bodies in such pathogenic bacteria as Salmonella Typhimurium and Shigella flexneri, which respectively cause gastroenteritis and diarrhea in humans ${ }^{49,50}$. In particular, we observed a similar timedependent appearance of the endogenous FtsZ in the lysate pellet of non-growing stationary-phase cells for either Salmonella Typhimurium SL1344 or Shigella flexneri serotype 2a 2457T (Fig. 6a). For each strain, we then observed a similar correlation between a higher degree of regrowth-delay body formation and a longer regrowth lag time (Fig. 6b and Supplementary Fig. S11) or a higher level of antibiotic tolerance (Fig. 6c). These observations again indicate that regrowth-delay body is generally formed in bacterial cells and its formation is correlated to the regrowth lag and antibiotic tolerance.

\section{Discussion}

Here, we reported our accidental discovery of a hitherto unreported bacterial subcellular structure that we designated as the regrowth-delay body. In retrospect, we made this revelation as a result of our initial in vivo protein photo-crosslinking and subsequent live-cell imaging analyses on FtsZ, not only with actively dividing cells (as have been extensively examined by others), but also with the non-dividing/non-growing cells (as have been rarely examined by others). We provided ample evidence to support our conclusion that the regrowth-delay bodies sequestering multiple key cellular proteins are formed in the non-growing cells and dissolved in re-growing cells, which in turn could be viewed as a marker for multidrugtolerant persisters. Considering multiple essential proteins sequestered in the regrowth-delay bodies, their dissolution might be a prerequisite for cell regrowth.

Regrowth-delay body represents a distinctive subcellular structure that allows the tiny subpopulation of persisters to be effectively identified in a large population of actively growing cells, a prerequisite for elucidating their physiological properties. Importantly, our findings imply that a bacterial persister is actually in a particular depth of persistence, as correlated with the status of its regrowthdelay bodies. In other words, a persister whose regrowthdelay bodies are to be dissolved rather effectively is in a shallow persistent state, thus to exhibit a relatively short regrowth lag whenever as an optimal growth condition becomes available. Conversely, a persister whose regrowth-delay bodies are to be maintained for an extended period of time even when an optimal growth condition becomes available is in a deep persistent state.

Having cells in different depths of persistence would conceivably allow certain number of persister cells to survive under any harmful condition. This explains how the formation of regrowth-delay bodies would provide an effective bet-hedging strategy for a bacterial species to maximize its possibility of survival in the highly unpredictable natural environment ${ }^{10,51,52}$. In a sense, the regrowth-delay bodies in a persister cell function as the biological timer that reflects the particular duration of regrowth lag for the non-growing bacterial cell to resume growth.

Our revelations also explain why the formation of persisters has long been viewed as a stochastic or heterogeneous phenomenon occurring in the bacterial cell populations $s^{53-56}$. This is mainly due to the high heterogeneity of regrowth-delay body formation in different individual cells as well as the progressive nature of their formation in each single cell. Because of this, a bacterial cell sample taken from different culturing point would be highly heterogeneous in regards of the status of the cellular regrowth-delay bodies or depth of persistence in different cells. It follows that the duration of regrowth lag, the level of drug tolerance, as well as the percentage of cells defined as persisters (by measuring the number of colony-forming units after treating an antibiotic) in the cell population, would most likely appear as inconsistent or stochastic values even in repeating experiments.

One difficulty in studying the persister cells is to unequivocally identify them, as they usually exist in extremely small numbers in a cell population that are actively growing ${ }^{8}$. The presence of the distinctive regrowth-delay bodies would prove to be greatly helpful in overcoming this difficulty (as exemplified by the data shown in Fig. 2b). This meanwhile may allow us to conduct single cell biochemistry and cell biology studies on persisters, including a characterization of the transcriptome, proteome and metabolome $\mathrm{e}^{10,57}$.

In light of our findings described here, the "viable but non-culturable" bacteria, which is known to evade the conventional culture-based microbiological detection ${ }^{11}$, may represent persister cells whose regrowth-delay bodies could not effectively dissolve under the commonly applied culturing conditions. After we learn more about the conditions that will effectively promote the dissolution of regrowth-delay bodies, we may be able to make these bacterial cells culturable under particular conditions. By the same token, in clinics, we might be able to find ways to eradicate the multidrug-tolerant recalcitrant pathogen 

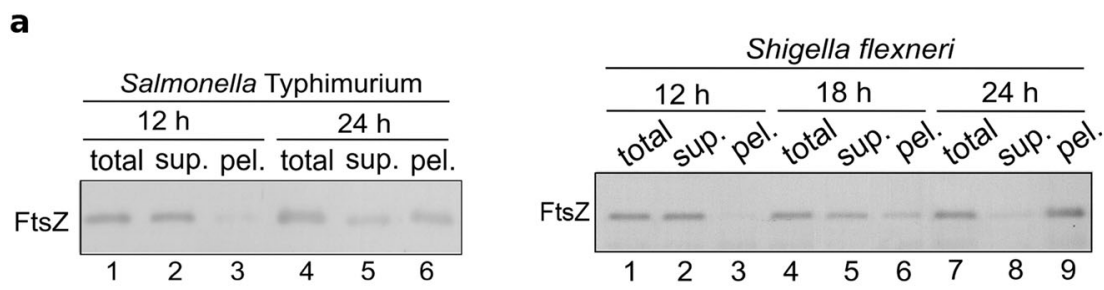

b
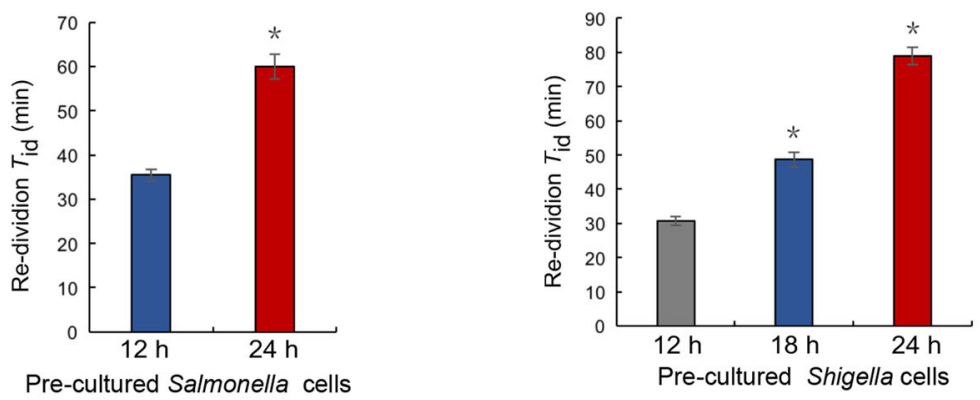

C
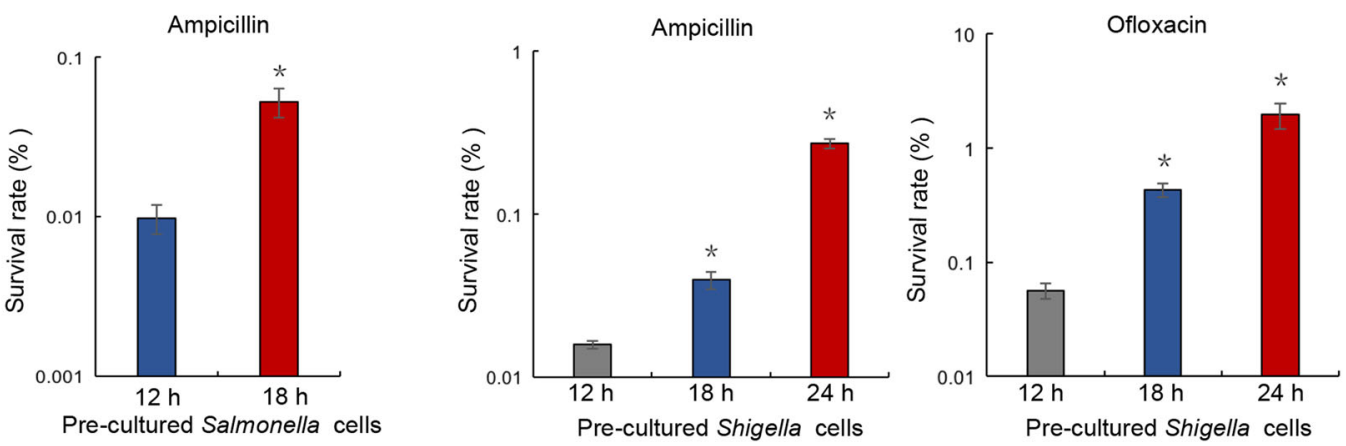

Fig. 6 Regrowth-delay bodies are also formed in the late stationary-phase cells of the pathogenic bacteria Salmonella Typhimurium SL1344 and Shigella flexneri serotype 2a 2457T. a Immunoblotting results for the detection of FtsZ in the indicated cell lysate fractions of the stationary-phase Salmonella Typhimurium or Shigella flexneri cells taken at the indicated time points, probed with antibodies against the E. coli FtsZ protein. $\mathbf{b}$ Re-division $T_{\text {id }}$ values of the Salmonella Typhimurium or Shigella flexneri cells that were pre-cultured to the indicated time points of the stationary-phase before being re-cultured in fresh LB medium. c Survival rates of the indicated re-cultured stationary-phase Salmonella Typhimurium or Shigella flexneri cells that were treated with the indicated antibiotics for $2 \mathrm{~h}$. The symbol ${ }^{*}$ in $\mathbf{b}$ and $\mathbf{c}$ denotes a significant difference between the compared pair of samples ( $P$-value $<0.05, t$-test). At least three biological replicates were analyzed for obtaining each value

persisters by promoting the dissolution of their regrowthdelay bodies in conjunction with an antibiotic treatment.

However, many questions remain unanswered concerning the biology of regrowth-delay bodies, as a new subcellular structure marking the non-growing persister bacterial cells. First, how are the components in the regrowth-delay bodies organized (to be revealed likely by high resolution electron microscopic analysis)? Second, what are the key signaling molecules that trigger their formation, and how are such signals sensed by cells? Third, how are the specifically sequestered proteins selected? Fourth, what signals trigger the regrowth-delay bodies to dissolve? Finally, do structures similar to regrowth-delay bodies exist in eukaryotes, especially those living as single-cell forms?

\section{Materials and methods}

Bacterial strains, plasmids, and genome modifications

Listed in Supplementary Table S1 are the genotypes of the used $E$. coli strains, all derived from the BW25113 strain with genotype: $F^{-}, \mathrm{DE}(\operatorname{araD}-\mathrm{araB}) 567$, lacZ4787(del)::rrnB-3, LAM ${ }^{-}$, rph-1, DE(rhaD-rhaB) 568, hsdR514. The analyzed pathogenic strains were Salmonella Typhimurium SL1344 and Shigella flexneri serotype 2a 2457T. All the plasmids employed in this study are listed in Supplementary Table S2. Genome modifications were performed using the $\lambda$-red genomic recombination system ${ }^{58}$. Newly generated plasmids and genome modifications were all confirmed by DNA sequencing. 


\section{Bacterial cell culturing}

LB liquid ( $10 \mathrm{~g} / \mathrm{l}$ tryptone, $5 \mathrm{~g} / \mathrm{l}$ yeast extract, and $5 \mathrm{~g} / \mathrm{l}$ $\mathrm{NaCl}$ ) and agar-containing solid culture medium were sterilized by autoclaving. Salmonella Typhimurium SL1344 and Shigella flexneri serotype 2a 2457T were cultured in LB medium with $30 \mu \mathrm{g} / \mathrm{ml}$ streptomycin. For plasmid selection, $50 \mu \mathrm{g} / \mathrm{ml}$ kanamycin, $34 \mu \mathrm{g} / \mathrm{ml}$ chloramphenicol, or $100 \mu \mathrm{g} / \mathrm{ml}$ ampicillin was added to the culturing medium. Log-phase and late stationary-phase cells refer to the cells that were cultured at $37^{\circ} \mathrm{C}$ in test tubes and shook at 260 r.p.m. for $6 \mathrm{~h}$ and $24 \mathrm{~h}$, respectively, after the overnight-cultured cells were diluted 100fold in fresh LB medium. The expression of CbtA or KilR was induced by addition of $0.2 \mu \mathrm{g} / \mathrm{ml}$ anhydrotetracycline. For membrane staining, FM4-64 $(2 \mu \mathrm{g} / \mathrm{ml})$ was added to the culturing medium, and the cells were then further cultured for another $1 \mathrm{~h}$.

In vivo protein photo-crosslinking of $\mathrm{pBpa}$ variants of FtsZ

To perform the photo-crosslinking analysis within the LY928-ftsZ-Avi strain (in which endogenous wild-type FtsZ protein was expressed with an Avi tag fused to its Cterminus) that we constructed, each $\mathrm{pBpa}$ variant was expressed from a plasmid at a level comparable with that of endogenous FtsZ, and the cells were cultured to log or late stationary phase at $37^{\circ} \mathrm{C}$ in $\mathrm{LB}$ medium containing $200 \mu \mathrm{M}$ pBpa. The cells were irradiated with UV light $(365 \mathrm{~nm})$ for $10 \mathrm{~min}$ at room temperature using a Hoefer UVC 500 Crosslinker (Amersham Biosciences, USA) and then collected by centrifugation at $13,000 \times g$ before being subjected to further (blotting) analysis.

\section{Fluorescence microscopic imaging}

Cell or cell lysate samples were placed on a glass dish (NEST Biotechnology, USA) and covered with agar before micrographs were acquired at $37^{\circ} \mathrm{C}$ (for the re-culturing cell samples) or $30^{\circ} \mathrm{C}$ (for all other samples) with an N-SIM imaging system (Nikon, Japan) using the 2D-SIM mode, a $\times 100 / 1.49$ NA oil-immersion objective (Nikon, Japan), and under excitation of a 488 or $561 \mathrm{~nm}$ laser beam. The 3D images were acquired with an N-SIM imaging system using the 3D mode. The samples were sectioned every $120 \mathrm{~nm}$ along the $Z$-axis. The images were further reconstructed using the NIS-Elements AR 4.20.00 (Nikon, Japan) before a further processing with the GNU image manipulation program. DV Elite microscope with specific optics was also utilized to perform live-cell imaging analysis. At least four images were obtained, and more than 50 bacterial cells were examined for each experiment. All experiments were biologically repeated at least three times.

\section{Cell lysate fractionations}

The non-growing late stationary-phase bacterial cells were prepared by growing the cells at $37^{\circ} \mathrm{C}$ (with shaking at 260 r.p.m.) for $24 \mathrm{~h}$ after the overnight-cultured cells were diluted 100-fold into fresh LB medium. The cell samples (such as those used in Supplementary Fig. S7) of the re-culturing experiments were prepared by transferring the 2-fold diluted non-growing late stationary-phase cells into fresh LB medium in the presence of chloramphenicol $(34 \mu \mathrm{g} / \mathrm{ml})$ and further culturing them at $37^{\circ}$ $\mathrm{C}$ (with shaking at 260 r.p.m.) to the indicated time points. The cells were then collected by centrifugation $(8000 \times g)$ and disrupted using a French press at $1000 \mathrm{MPa}$ before centrifugation at $13,000 \times g$ to separate the supernatant and pellet fractions.

\section{Protein purification and mass spectrometry analysis}

The photo-crosslinked products of pBpa variants of FtsZ-Avi generated in the LY928 strain were individually purified using streptavidin magnetic beads after the pellet containing the photo-crosslinked products was dissolved in $8 \mathrm{M}$ urea and diluted 10-fold in binding buffer. The eluted protein samples were then further resolved by SDS-PAGE. For identification of proteins in the regrowthdelay bodies, the pellet from late stationary-phase wildtype cell lysates was collected, dissolved in $8 \mathrm{M}$ urea, and centrifuged again at $13,000 \times g$ before removing the new pellet. The supernatant was then concentrated 10-fold and resolved by SDS-PAGE. In both of the above cases, the protein bands of SDS-PAGE that could be clearly visualized by Coomassie blue staining on the gel, and were excised and sent for mass spectrometry analysis.

\section{Blotting analysis}

Each sample, including the cell lysate, supernatant fraction, pellet fraction, or UV-irradiated cells, was supplemented with the sample buffer, boiled, and resolved via tricine SDS-PAGE before being further probed with particular antibodies or streptavidin-AP conjugate (for the Avi-tagged proteins) for the blotting analysis. The protein bands visualized on the gels were scanned and processed using the GNU image manipulation program.

\section{CRISPRi experiments}

CRISPRi was performed according to previously reported methods ${ }^{48}$. Briefly, plasmids carrying a crRNA that targets the nuoA or $s d h C$ gene were transformed into $E$. coli cells in which the proteins for recognizing and binding specific DNA sequences were expressed from the Cascade operon while the gene (cas3 gene) encoding the protein that cleaves the target sequence was deleted. The DNA sequences designed for knocking down the nuoA and the $s d h C$ genes were: ATAGCGAATGCCCAGTGATGAGCGATGACTTC and AATGTGAAAAAACA AAGACCTGTTAATCTGGA, respectively. The control plasmid carried a non-targeting crRNA sequence: CTGCTGGAGCTGGCTG CAAGGCAAGCCGCCCA. 
The crRNAs on the plasmids were transcribed constitutively rather than induced.

\section{Cell regrowth and calculation of the average re-division $T_{\mathrm{id}}$}

Log-phase or late stationary-phase cells of a particular type were diluted 40-fold into fresh LB medium and cultured at $37^{\circ} \mathrm{C}$ with shaking (260 r.p.m.). Growth curves were prepared by measuring the $\mathrm{OD}_{600}$ value of the cultured cells at $30-\mathrm{min}$ intervals. The re-division $T_{\text {id }}$ value was calculated as $30 / \log _{2}^{N t 1 / N t 0} \min$, where $\mathrm{N}_{\mathrm{t} 0}$ and $\mathrm{N}_{\mathrm{t} 1}$ were the numbers of cells at 0 and $30 \mathrm{~min}$, respectively. The $\mathrm{N}_{\mathrm{t} 1} / \mathrm{N}_{\mathrm{to}}$ ratio for each batch of cultured cells was calculated based on the increase in optical density at 600 $\mathrm{nm}$ (the correlation between the cell number and the $\mathrm{OD}_{600}$ value was determined by preparing a standard curve). At least three biological replicates were analyzed for obtaining each value.

\section{Assay for cell survival after antibiotic treatment}

Stationary-phase cells were diluted 40-fold into fresh LB medium containing either $5 \mu \mathrm{g} / \mathrm{ml}$ ofloxacin or $200 \mu \mathrm{g} / \mathrm{ml}$ ampicillin and incubated at $37^{\circ} \mathrm{C}$ with shaking (260 r.p. $\mathrm{m}$.) for $2 \mathrm{~h}$. The cells were then collected by centrifugation (to remove the culture medium and the antibiotics), resuspended in phosphate-buffered saline (PBS), and serially diluted in PBS buffer before being spotted on LB agar plates for CFU counting. The cell survival rate was calculated as follows: (number of colonies formed after antibiotic treatment)/(number of colonies formed without antibiotic treatment $) \times 100$. At least three biological replicates were analyzed for obtaining each value.

\section{Acknowledgements \\ We thank Professor Harold Erickson (Duke University, USA) for providing us the pJSB100 plasmid, and Professor Peter Schultz (The Scripps Research Institute, USA) for providing us with the plasmids that carry the genes encoding the orthogonal tRNA and orthogonal amino acyl-tRNA for pBpa incorporation. We thank Keio Collections for providing us the wild-type E. coli strain. We thank Dr. Xiaoyun Liu (Peking University, China) for providing the Salmonella \\ Typhimurium and Shigella flexneri strains. We thank the Core Facilities at the School of Life Sciences, Peking University, for assistance using the structured illumination microscope (SIM) and DV Elite microscope, and we are grateful to Dr. Chunyan Shan and Dr. Xiaochen Li for assisting us with the fluorescence microscopic imaging analysis. We thank Dr. Wen Zhou at the Mass Spectrometry Facility of the National Center for Protein Sciences at Peking University for assistance on the mass spectrometry analysis. We thank Professor Chong Liu from Zhejiang University and Professor Xinmiao Fu from Fujian Normal University for useful discussions. This work was supported by funds from the National Natural Science Foundation of China (No. 31670775 and 31470766 to Z.Y.C.), the National Basic Research Program of China (No. 2012 CB917300 to Z.Y.C.), and the Qidong-SLS Innovation Fund.}

\section{Author contributions}

J.Y. and Y.L. designed and performed the major experiments, analyzed the data, and drafted the manuscript. H.Y. designed and performed part of the experiments. Z.C. supervised the entirety of the study.

Conflict of interest

The authors declare that they have no conflict of interest.

\section{Publisher's note}

Springer Nature remains neutral with regard to jurisdictional claims in published maps and institutional affiliations.

Supplementary Information accompanies the paper at (https://doi.org/ 10.1038/541421-019-0080-3).

Received: 27 November 2018 Revised: 28 December 2018 Accepted: 1 January 2019

Published online: 22 January 2019

\section{References}

1. Burke, V., Sprague, A. \& Barnes, L. V. Dormancy in bacteria. J. Infect. Dis. 36, 555-560 (1925).

2. Chesney, A. M. The latent period in the growth of bacteria. J. Exp. Med. 24, 387-418 (1916).

3. Kaprelyants, A. S., Gottschal, J. C. \& Kell, D. B. Dormancy in non-sporulating bacteria. FEMS Microbiol. Rev. 10, 271-285 (1993).

4. Lewis, K. Persister cells, dormancy and infectious disease. Nat. Rev. Microbiol. 5, 48-56 (2007).

5. Lewis, K. Persister cells. Annu. Rev. Microbiol. 64, 357-372 (2010).

6. Monod, J. The growth of bacterial cultures. Annu. Rev. Microbiol. 3, 371-394 (1949).

7. Roszak, D. B. \& Colwell, R. R. Survival strategies of bacteria in the natural environment. Microbiol. Rev. 51, 365-379 (1987).

8. Balaban, N. Q., Gerdes, K., Lewis, K. \& McKinney, J. D. A problem of persistence: still more questions than answers? Nat. Rev. Microbiol. 11, 587-591 (2013).

9. Kaldalu, N., Hauryliuk, V. \& Tenson, T. Persisters-as elusive as ever. Appl. Microbiol. Biotechnol. 100, 6545-6553 (2016).

10. Kell, D., Potgieter, M. \& Pretorius, E. Individuality, phenotypic differentiation, dormancy and 'persistence' in culturable bacterial systems: commonalities shared by environmental, laboratory, and clinical microbiology. F1000Res. 4 179 (2015).

11. Pinto, D., Santos, M. A. \& Chambel, L. Thirty years of viable but nonculturable state research: unsolved molecular mechanisms. Crit. Rev. Microbiol. 41, 61-76 (2015).

12. Coplans, M. Influences affecting the growth of microorganisms-latency: inhibition: mass action. J. Pathol. Bacteriol. 14, 1-27 (1910).

13. Rolfe, M. D. et al. Lag phase is a distinct growth phase that prepares bacteria for exponential growth and involves transient metal accumulation. J. Bacteriol. 194, 686-701 (2012).

14. Bigger, J. Treatment of Staphylococcal infections with penicillin by intermittent sterilisation. Lancet 244, 497-500 (1944).

15. Fisher, R. A., Gollan, B. \& Helaine, S. Persistent bacterial infections and persister cells. Nat. Rev. Microbiol. 15, nrmicro.2017.42 (2017).

16. Mcdermott, W. Microbial persistence. Yale J. Biol. Med. 30, 257-291 (1958)..

17. Ayrapetyan, M., Williams, T. C. \& Oliver, J. D. Bridging the gap between viable but non-culturable and antibiotic persistent bacteria. Trends Microbiol. 23, 7-13 (2015).

18. $\mathrm{Xu}, \mathrm{H}$. S. et al. Survival and viability of nonculturable Escherichia coli and Vibrio cholerae in the estuarine and marine environment. Microb. Ecol. 8, 313-323 (1982).

19. Black, D. S., Kelly, A. J., Mardis, M. J. \& Moyed, H. S. Structure and organization of hip, an operon that affects lethality due to inhibition of peptidoglycan or DNA synthesis. J. Bacteriol. 173, 5732-5739 (1991).

20. Black, D. S., Irwin, B. \& Moyed, H. S. Autoregulation of hip, an operon that affects lethality due to inhibition of peptidoglycan or DNA synthesis. J. Bacteriol. 176, 4081-4091 (1994).

21. Moyed, H. S. \& Bertrand, K. P. hipA, a newly recognized gene of Escherichia coli K-12 that affects frequency of persistence after inhibition of murein synthesis. J. Bacteriol. 155, 768-775 (1983).

22. Korch, S. B., Henderson, T. A. \& Hill, T. M. Characterization of the hipA7 allele of Escherichia coli and evidence that high persistence is governed by (p)ppGpp synthesis: persistence and (p)ppGpp synthesis in E. coli. Mol. Microbiol. 50, 1199-1213 (2003)

23. Chowdhury, N., Kwan, B. W. \& Wood, T. K. Persistence increases in the absence of the alarmone guanosine tetraphosphate by reducing cell growth. Sci. Rep. 6, 20519 (2016). 
24. Orman, M. A. \& Brynildsen, M. P. Inhibition of stationary phase respiration impairs persister formation in E. coli. Nat. Commun. 6, 7983 (2015).

25. Balaban, N. Q., Merrin, J., Chait, R., Kowalik, L. \& Leibler, S. Bacterial persistence as a phenotypic switch. Science 305, 1622-1625 (2004).

26. Fu, X., Shi, X., Yan, L., Zhang, H. \& Chang, Z. In vivo substrate diversity and preference of small heat shock protein $\mathrm{lbpB}$ as revealed by using a genetically incorporated photo-cross-linker. J. Biol. Chem. 288, 31646-31654 (2013).

27. Zhang, $M$. et al. A genetically incorporated crosslinker reveals chaperone cooperation in acid resistance. Nat. Chem. Biol. 7, 671-677 (2011).

28. Dai, K. \& Lutkenhaus, J. ftsZ is an essential cell division gene in Escherichia coli. J. Bacteriol. 173, 3500-3506 (1991).

29. Erickson, H. P., Anderson, D. E. \& Osawa, M. FtsZ in bacterial cytokinesis: cytoskeleton and force generator all in one. Microbiol. Mol. Biol. Rev. 74, 504-528 (2010).

30. Haeusser, D. P. \& Margolin, W. Splitsville: structural and functional insights into the dynamic bacterial Z ring. Nat. Rev. Microbiol. 14, 305-319 (2016).

31. Beech, P. L. et al. Mitochondrial FtsZ in a chromophyte alga. Science 287, 1276-1279 (2000).

32. TerBush, A. D., Yoshida, Y. \& Osteryoung, K. W. FtsZ in chloroplast division: structure, function and evolution. Curr. Opin. Cell Biol. 25, 461-470 (2013).

33. Guan, F. et al. Lateral interactions between protofilaments of the bacterial tubulin homolog FtsZ are essential for cell division. elife 7, pii: e35578 (2018).

34. Chin, J. W., Martin, A. B., King, D. S., Wang, L. \& Schultz, P. G. Addition of a photocrosslinking amino acid to the genetic code of Escherichia coli. Proc. Natl Acad. Sci. USA 99, 11020-11024 (2002).

35. Ma, X., Ehrhardt, D. W. \& Margolin, W. Colocalization of cell division proteins FtsZ and FtsA to cytoskeletal structures in living Escherichia coli cells by using green fluorescent protein. Proc. Natl Acad. Sci. USA 93 12998-13003 (1996).

36. Stricker, J. \& Erickson, H. P. In vivo characterization of Escherichia coli ftsZ mutants: effects on Z-ring structure and function. J. Bacteriol. 185, 4796-4805 (2003).

37. Fishov, I. \& Woldringh, C. L. Visualization of membrane domains in Escherichia coli. Mol. Microbiol. 32, 1166-1172 (1999).

38. Lindner, A. B., Madden, R., Demarez, A., Stewart, E. J. \& Taddei, F. Asymmetric segregation of protein aggregates is associated with cellular aging and rejuvenation. Proc. Natl Acad. Sci. USA 105, 3076-3081 (2008).

39. Winkler, J. et al. Quantitative and spatio-temporal features of protein aggregation in Escherichia coli and consequences on protein quality control and cellular ageing. EMBO J. 29, 910-923 (2010).

40. Maisonneuve, E., Ezraty, B. \& Dukan, S. Protein aggregates: an aging factor involved in cell death. J. Bacteriol. 190, 6070-6075 (2008).
41. Kwiatkowska, J., Matuszewska, E., Kuczyńska-Wiśnik, D. \& Laskowska, E. Aggregation of Escherichia coli proteins during stationary phase depends on glucose and oxygen availability. Res. Microbiol. 159, 651-657 (2008).

42. Leszczynska, D., Matuszewska, E., Kuczynska-Wisnik, D., Furmanek-Blaszk, B. \& Laskowska, E. The formation of persister cells in stationary-phase cultures of Escherichia coli is associated with the aggregation of endogenous proteins. PLoS One 8, e54737 (2013).

43. Mukherjee, A. \& Lutkenhaus, J. Purification, assembly, and localization of FtsZ. Methods Enzymol. 298, 296-305 (1998).

44. Conter, A., Bouché, J. P. \& Dassain, M. Identification of a new inhibitor of essential division gene ftsZ as the kil gene of defective prophage Rac. J. Bacteriol. 178, 5100-5104 (1996).

45. Heller, D. M., Tavag, M. \& Hochschild, A. CbtA toxin of Escherichia coli inhibits cell division and cell elongation via direct and independent interactions with FtsZ and MreB. PLoS Genet. 13, e1007007 (2017)

46. Löwe, J. \& Amos, L. A. Crystal structure of the bacterial cell-division protein FtsZ. Nature 391, 203-206 (1998).

47. Zhou, Y. \& Gottesman, S. Regulation of proteolysis of the stationary-phase sigma factor RpoS. J. Bacteriol. 180, 1154-1158 (1998).

48. Luo, M. L., Mullis, A. S., Leenay, R. T. \& Beisel, C. L. Repurposing endogenous type I CRISPR-Cas systems for programmable gene repression. Nucleic Acids Res. 43, 674-681 (2015).

49. Jennison, A. V. \& Verma, N. K. Shigella flexneri infection: pathogenesis and vaccine development. FEMS Microbiol. Rev. 28, 43-58 (2004).

50. Graham, S. M. Salmonellosis in children in developing and developed countries and populations. Curr. Opin. Infect. Dis. 15, 507-512 (2002).

51. Maisonneuve, E. \& Gerdes, K. Molecular mechanisms underlying bacterial persisters. Cell 157, 539-548 (2014).

52. Veening, J.-W., Smits, W. K. \& Kuipers, O. P. Bistability, epigenetics, and Bethedging in bacteria. Annu. Rev. Microbiol. 62, 193-210 (2008).

53. Allison, K. R., Brynildsen, M. P. \& Collins, J. J. Heterogeneous bacterial persisters and engineering approaches to eliminate them. Curr. Opin. Microbiol. 14 593-598 (2011).

54. Gefen, O. \& Balaban, N. Q. The importance of being persistent: heterogeneity of bacterial populations under antibiotic stress. FEMS Microbiol. Rev. 33, 704-717 (2009).

55. Amato, S. M. \& Brynildsen, M. P. Persister heterogeneity arising from a single metabolic stress. Curr. Biol. 25, 2090-2098 (2015).

56. Dhar, N. \& McKinney, J. D. Microbial phenotypic heterogeneity and antibiotic tolerance. Curr. Opin. Microbiol. 10, 30-38 (2007).

57. Taniguchi, Y. et al. Quantifying E. coli proteome and transcriptome with singlemolecule sensitivity in single cells. Science 329, 8 (2010).

58. Lee, D. J. et al. Gene doctoring: a method for recombineering in laboratory and pathogenic Escherichia coli strains. BMC Microbiol. 9, 252 (2009). 